\title{
Article \\ Catalytic Effect of Ti or Pt in a Hexagonal Boron Nitride Surface for Capturing $\mathrm{CO}_{2}$
}

\author{
J. M. Ramirez-de-Arellano ${ }^{1, *(\mathbb{D})}$, A. Fransuani Jiménez G. ${ }^{2}$ (D) and L. F. Magaña ${ }^{2, *(D)}$ \\ 1 Tecnologico de Monterrey, Escuela de Ingeniería y Ciencias, Av. Eugenio Garza Sada 2501, \\ Monterrey 64849, Mexico \\ 2 Instituto de Física, Universidad Nacional Autónoma de México, Apartado Postal 20-364, \\ Ciudad de México 04510, Mexico; fransuani@estudiantes.fisica.unam.mx \\ * Correspondence: jramirezdearellano@tec.mx (J.M.R.-d.-A.); fernando@fisica.unam.mx (L.F.M.)
}

Citation: Ramirez-de-Arellano, J.M.; Jiménez G., A.F.; Magaña, L.F. Catalytic Effect of Ti or Pt in a Hexagonal Boron Nitride Surface for Capturing $\mathrm{CO}_{2}$. Crystals 2021, 11, 662 https://doi.org/10.3390/cryst11060662

Academic Editor: Radu Custelcean

Received: 18 May 2021

Accepted: 4 June 2021

Published: 10 June 2021

Publisher's Note: MDPI stays neutral with regard to jurisdictional claims in published maps and institutional affiliations.

Copyright: (C) 2021 by the authors Licensee MDPI, Basel, Switzerland. This article is an open access article distributed under the terms and conditions of the Creative Commons Attribution (CC BY) license (https:// creativecommons.org/licenses/by/ $4.0 /)$.

\begin{abstract}
We investigated the effect of doping a hexagonal boron nitride surface (hBN) with Ti or Pt on the adsorption of $\mathrm{CO}_{2}$. We performed first-principles molecular dynamics simulations (FPMD) at atmospheric pressure, and $300 \mathrm{~K}$. Pristine hBN shows no interaction with the $\mathrm{CO}_{2}$ molecule. We allowed the $\mathrm{Ti}$ and $\mathrm{Pt}$ atoms to interact separately, with either a B-vacancy or an $\mathrm{N}$-vacancy. Both $\mathrm{Ti}$ and Pt ended chemisorbed on the surface. The system hBN + Ti always chemisorbed the $\mathrm{CO}_{2}$ molecule. This chemisorption happens in two possible ways. One is without dissociation, and in the other, the molecule breaks in $\mathrm{CO}$ and $\mathrm{O}$. However, in the case of the Pt atom as dopant, the resulting system repels the $\mathrm{CO}_{2}$ molecule.
\end{abstract}

Keywords: $\mathrm{CO}_{2}$ absorption; ab-initio; DFT calculations; hBN surface

\section{Introduction}

Hexagonal boron nitride (hBN) is part of the layered, 2D van der Waals materials similar to graphene. It is an ultrathin $\mathrm{sp}^{2}$-hybridized material. The B-N bond is only $1.8 \%$ larger than the $\mathrm{C}-\mathrm{C}$ bond in graphene. The properties of the surface of hexagonal boron nitride are a feature of interest in many applications. It is very stable chemically, not easy to oxidize. It presents a bandgap of around $5.971 \mathrm{eV}$ [1]. This compound has excellent optical [1,2] and mechanical [3] properties, high thermal conductivity, and chemical inertness. It has raised interest about its capability of adsorption of some pollutant gases, like $\mathrm{CO}_{2}$.

Carbon dioxide is a greenhouse gas that contributes heavily to climate change. There is a worldwide interest in capturing $\mathrm{CO}_{2}$ molecules. Many researchers focus their efforts on finding ways of absorbing it and potentially using it as a source of energy to reduce the polluting effects this gas has on the environment [4].

Several studies have been made on using BN-based systems for the capture of $\mathrm{CO}_{2}$. For instance, boron-enriched boron nitride nanotubes (BNNT) can chemisorb $\mathrm{CO}_{2}$ at ambient conditions via a boron antisite BN [5]. Furthermore, some reports show with DFT studies that boron fullerenes with an encapsulated $\mathrm{Li}$ atom $\left(\mathrm{Li} @ \mathrm{~B}_{40}\right)$ adsorb a $\mathrm{CO}_{2}$ molecule. The Li atom acts as a catalyst in this case [6]. The capabilities to absorb $\mathrm{CO}_{2}$ increase with changes in the charge state of pristine hBN sheets, BN nanotubes, and BN fullerenes [7,8]; besides, investigations on different $\mathrm{hBN}$ nanomaterials like foams and porous $\mathrm{BN}(h p \mathrm{BN})$ explored their $\mathrm{CO}_{2}$ absorption capabilities as well $[9,10]$.

Considering these studies, we explore a possible option for $\mathrm{CO}_{2}$ absorption using boron nitride, titanium, and platinum. The last two are transition metals commonly used for varied industrial applications. We study the role of $\mathrm{Ti}$ and $\mathrm{Pt}$ as catalysts for the capture of $\mathrm{CO}_{2}$. The results of this work can help enlighten the understanding of the mechanisms of $\mathrm{CO}_{2}$ capture for environmental uses. 


\section{Materials and Methods}

First-principles molecular dynamics (FPMD) calculations were performed, using the density functional theory (DFT) and the pseudopotential formalism [11,12]. We used the Quantum ESPRESSO suite code for all the simulations [13,14] and Xcrysden for visualization [15]. The pseudopotentials used are of the norm-conserving Troullier-Martins [16], projector augmented wave (PAW) type [17], in the entirely separable Kleinman-Bylander form [18]. We used the Perdew-Burke-Ernzerhof (PBE) approximation for the generalized gradient approximation (GGA) [19]. The calculations were non-spin polarized and non-relativistic, as our system is in general non-magnetic. The spin-polarization should be considered when there is an interest on its band structure or the magnetic properties, which is not the case for this work. Additionally, it has been shown that spin-polarization does not affect the binding energies [20].

We took as valence electronic states for boron: $2 s^{2} 2 p^{1}$; for nitrogen: $2 s^{2} 2 p^{3}$; for titanium: $3 s^{2} 3 p^{6} 3 d^{2} 4 s^{2}$; for platinum: $6 s^{1} 5 d^{9}$; for carbon: $2 s^{2} 2 p^{2}$ and for oxygen: $2 s^{2} 2 p^{4}$. The k-point mesh consisted of $40 \mathrm{k}$ points within the Monkhorst-Pack scheme [21]. The energy cutoff was $100 \mathrm{Ry}$ or around $1360 \mathrm{eV}$. Velocity rescaling was used for the temperature control in the FPMD calculations, which were performed at $300 \mathrm{~K}$.

The system consists of an hBN hexagonal unit cell containing 50 atoms when pristine, using periodic boundary conditions. The cell parameters are $\mathrm{a}=\mathrm{b}=12.49 \AA$ and $\mathrm{c}=26.39 \AA$, the latter being large enough to avoid spurious interactions between repetitions of the cell along the c direction. We considered two different cases: (a) a B vacancy and (b) an N vacancy (Figure 1). We explored the interaction between a Ti atom or a Pt atom and the system with a vacancy. Afterward, we calculated the interaction between the resulting systems and $\mathrm{COO}_{2}$ molecule.

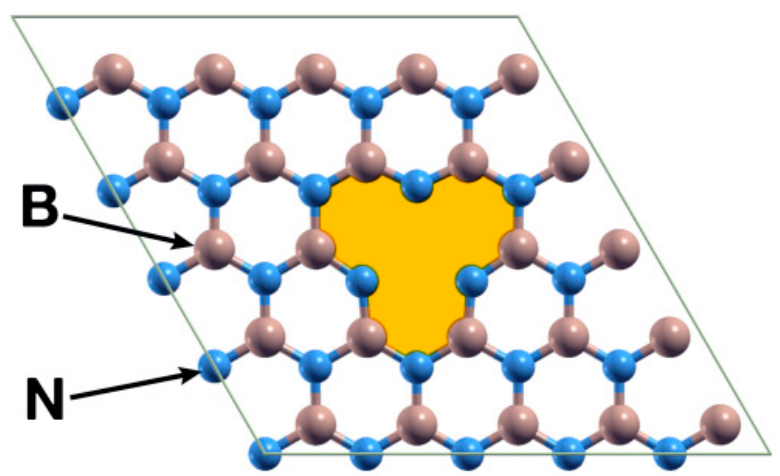

(a)

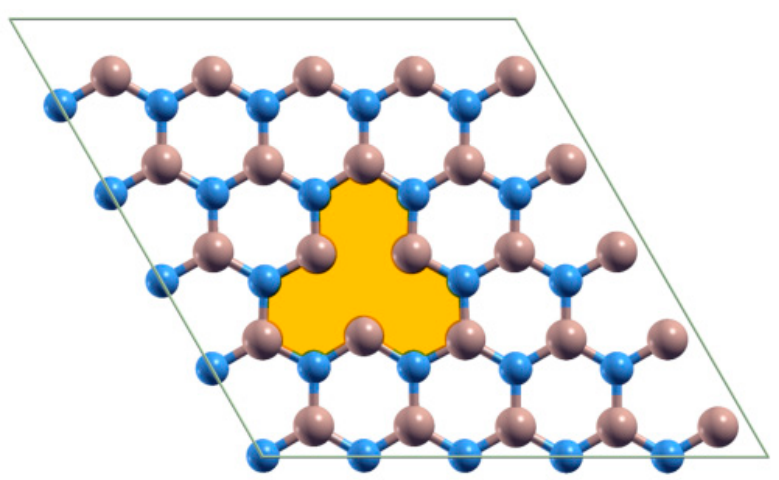

(b)

Figure 1. The two vacancies considered for the BN unit cell, originally containing 50 atoms: (a) The hBN surface with a boron atom removed; (b) The same surface but with a nitrogen atom removed. The figure presents the structures after a relaxation. We include the shaded areas for visualization purposes.

We calculated the absorption energies using the following formula:

$$
\mathrm{E}_{\mathrm{ads}}=\mathrm{E}_{\mathrm{system} 1+\text { system } 2}-\mathrm{E}_{\mathrm{system} 1}-\mathrm{E}_{\mathrm{system} 2}
$$

The term $\mathrm{E}_{\text {system1+system2 }}$ is the average total energy of the combined system after the FPMD calculation run. The following two terms refer to the average total energy of the separate systems after a similar calculation is run on each of them separately. The border between physisorption and chemisorption is not an exact one, but it is usually considered to be around $0.5 \mathrm{eV}$ per molecule. This corresponds to binding energies not much larger than $100 \mathrm{meV}$ [22]. 


\section{Results}

The pseudopotentials were tested and validated by performing a structural relaxation of the corresponding structures. For instance, with the pseudopotentials for $\mathrm{B}$ and $\mathrm{N}$, we calculated the B-N bond length in the hBN surface, obtaining a value of $1.447 \AA$, compared with the experimental value of $1.446 \AA$. For the lattice parameter c, we got $6.6607 \AA$, compared to the reported observed value of $6.661 \AA$ [23]. In the case of the $\mathrm{CO}_{2}$ molecule, we obtained a C-O bond length of $1.1708 \AA$ and $1.1710 \AA$, and an angle of $179.999^{\circ}$, compared with the experimental values of $1.163 \AA$ and $180^{\circ}$, respectively [24]. For the $\alpha$-Ti phase, we calculated a cell parameter of $2.931 \AA$, compared with the value of $2.9064 \AA$ reported in the literature [25]. For platinum, we got $4.077 \AA$ compared to the experimental value of $3.9218 \AA$ [26].

After an FPMD calculation, we obtained that the pristine $\mathrm{hBN}$ surface does not interact with a $\mathrm{CO}_{2}$ molecule. The same occurred when we removed either one boron atom or one nitrogen atom to create a vacancy. This result agrees with previous studies [4]. Figure 2 shows the PDOS for the pristine 50-atoms hBN cell. Notice the hybridization of states $s$ and $p$ of boron and nitrogen to form the hBN surface. The B-N bond results from the $s p 2$ hybridization, like in graphene atoms.

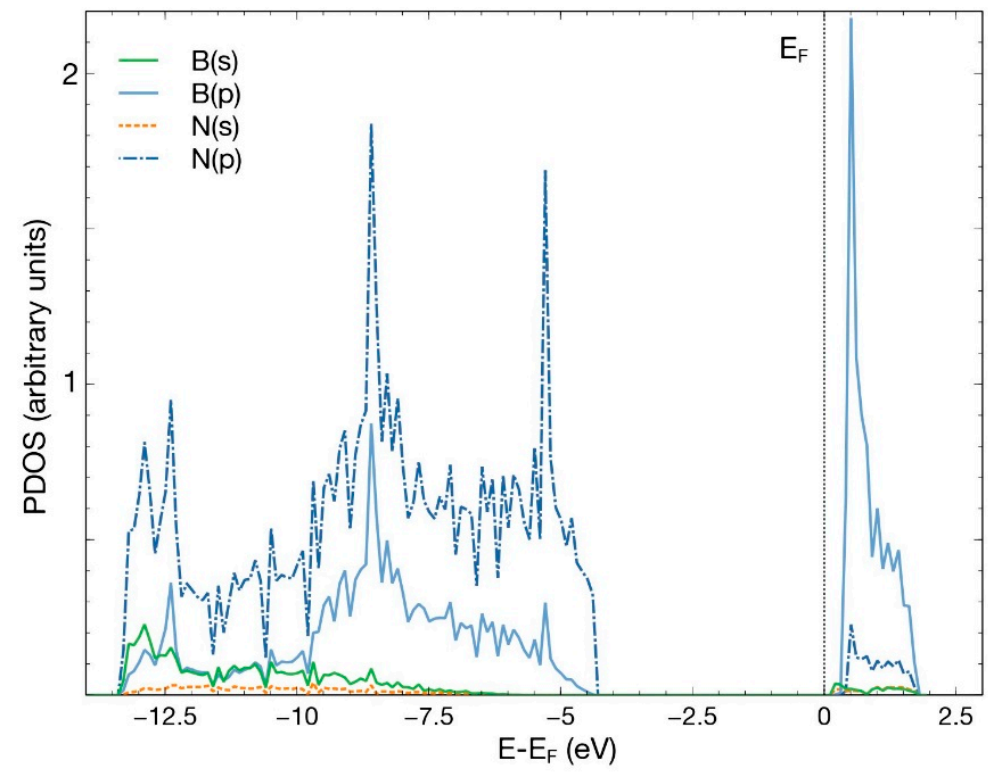

Figure 2. PDOS for the pristine hBN surface, consisting of 50 atoms. The hybridization of states $s$ and $p$ of boron and nitrogen is evident.

\section{1. hBN Surface with a B Vacancy}

To explore different ways of catalyzing the absorption of the $\mathrm{CO}_{2}$ molecule, we considered a $\mathrm{Ti}$ atom or a $\mathrm{Pt}$ atom as candidates to occupy the vacancy created after removing a B atom (Figure 1a). Figure 3 presents the PDOS of this system. From this figure, we notice the hybridization of orbitals $s$ and $p$, with a minor contribution from orbitals $s$ from boron and nitrogen. 


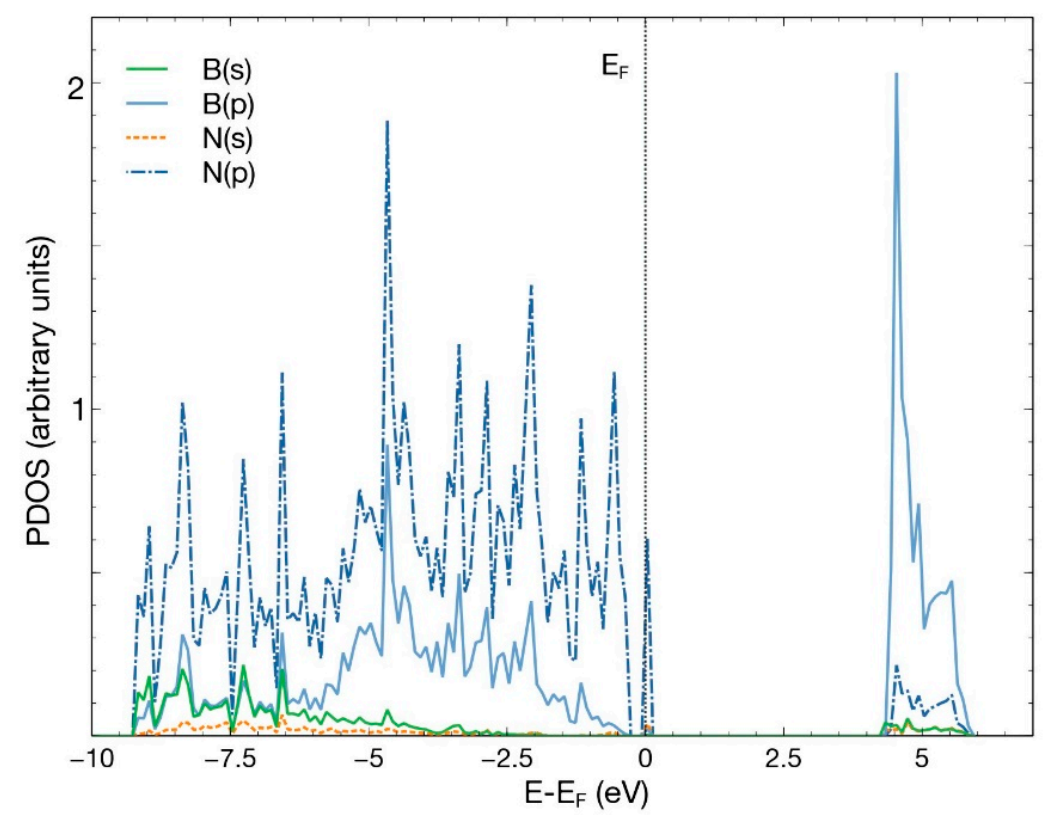

Figure 3. PDOS for the hBN surface with a B vacancy. The hybridization of orbitals $s$ and $p$ is evident, with a decrease in $s$ orbitals from boron and nitrogen.

\subsubsection{Ti Absorption on a B Vacancy}

The Ti atom was the first to be studied in this system. We placed a Ti atom $2.5 \AA$ above the surface, directly over the vacancy site. After 547 steps, the FPMD calculation shows at $300 \mathrm{~K}$ and atmospheric pressure that the Ti atom chemisorbs with an absorption energy of $-11.39 \mathrm{eV}$. Figure 4 shows the initial and final configuration for this case. A Löwdin charge analysis shows that the Ti atom transfers 1.03 electrons to the surface, particularly to the nitrogen atoms surrounding it. Figure 5 shows the PDOS for the titanium atom absorbed in a B vacancy site on the $h B N$ surface.
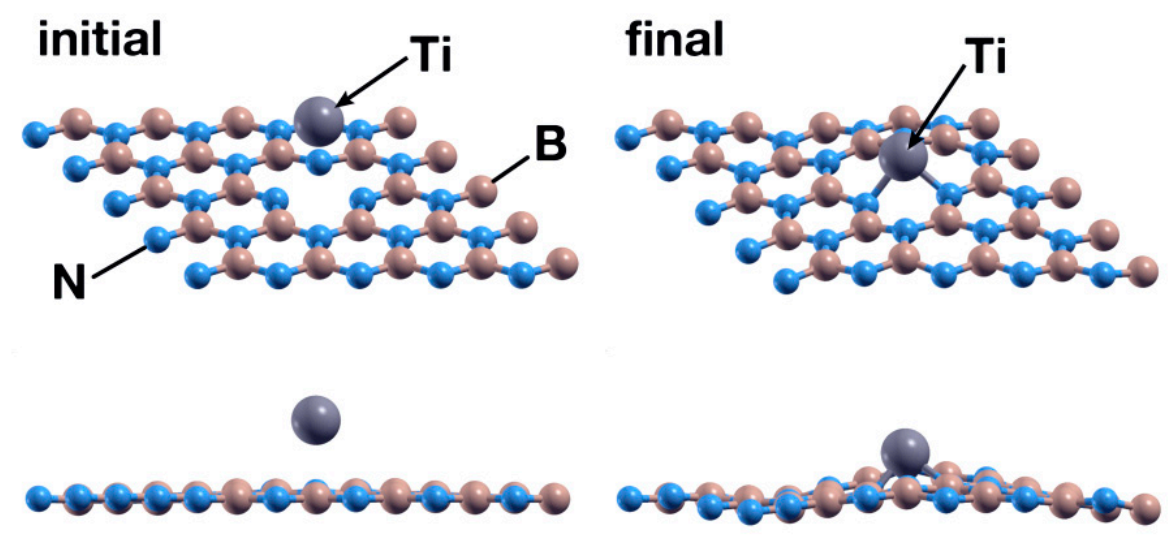

Figure 4. Initial (left) and final (right) configurations of the FPMD calculation for the first case considered. The upper images show a perspective view of the system, while the lower images show a picture of the $\mathrm{XZ}$ plane. The titanium atom is chemisorbed by the hBN surface with a B vacancy, occupying the vacancy. 


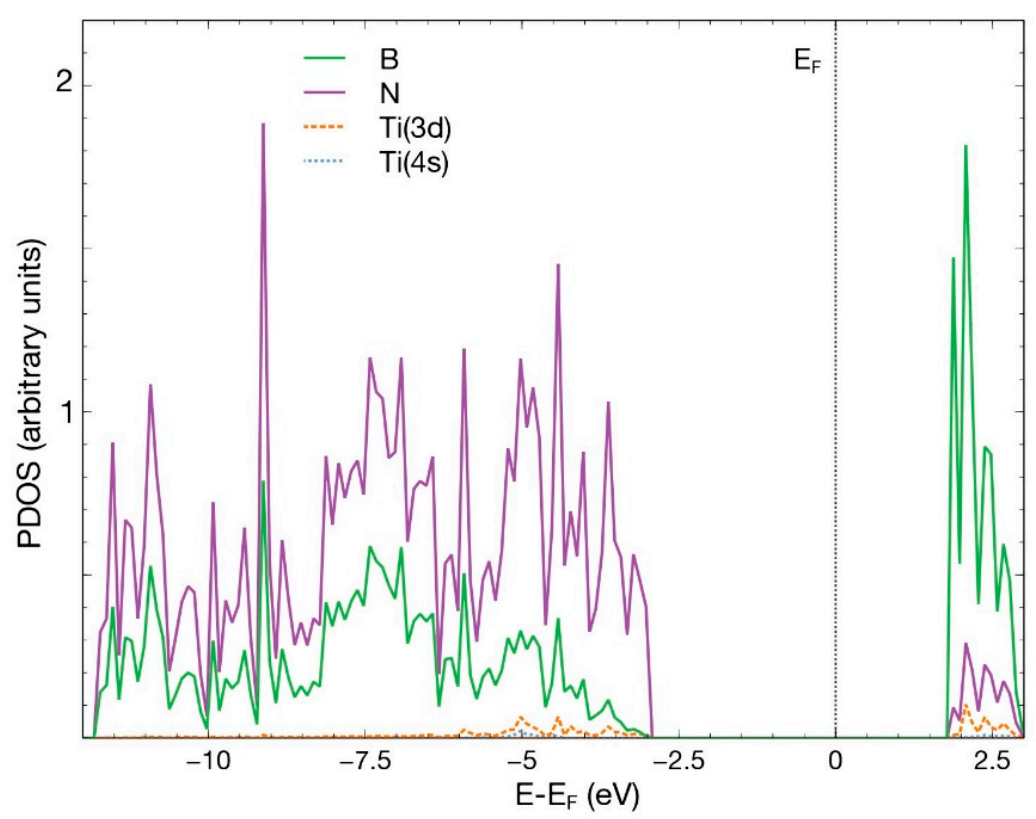

Figure 5. PDOS for the titanium atom absorbed in a B vacancy site on the hBN surface. This figure shows the $p$ orbitals for boron and nitrogen and the $s$ and $\boldsymbol{d}$ orbitals for Ti.

Figure 5 displays the $p$ orbitals for boron and nitrogen, and the $s$ and $\boldsymbol{d}$ orbitals for Ti. For energies below $-2.5 \mathrm{eV}$, notice the hybridization of the $p$ orbitals of boron and nitrogen and the titanium $4 s$ and $3 d$ orbitals. The contribution of the titanium orbitals is rather small. A similar behavior occurs for energies above $1.5 \mathrm{eV}$. Some states appear around the Fermi energy.

\subsection{2. $\mathrm{CO}_{2}$ Absorption on an $\mathrm{hBN}$ Surface + Ti Absorbed in a B Vacancy}

Taking the previous case as the starting point, we now placed a $\mathrm{CO}_{2}$ molecule $3 \AA$ above the Ti atom. The carbon dioxide molecule is linear, and it was oriented parallel to the surface. We obtained that the Ti atom traps the $\mathrm{CO}_{2}$ molecule via an oxygen atom (Figure 6). The adsorption energy is $-1.04 \mathrm{eV}$. Besides, the angle $\mathrm{O}-\mathrm{C}=\mathrm{O}$ changes from $180^{\circ}$ to $141.2^{\circ}$. However, we found null interaction with the pristine $\mathrm{hBN}$ and with $\mathrm{hBN}$ with vacancies. The Ti atom donates 0.37 to the $\mathrm{CO}_{2}$ molecule in this process of chemisorption.

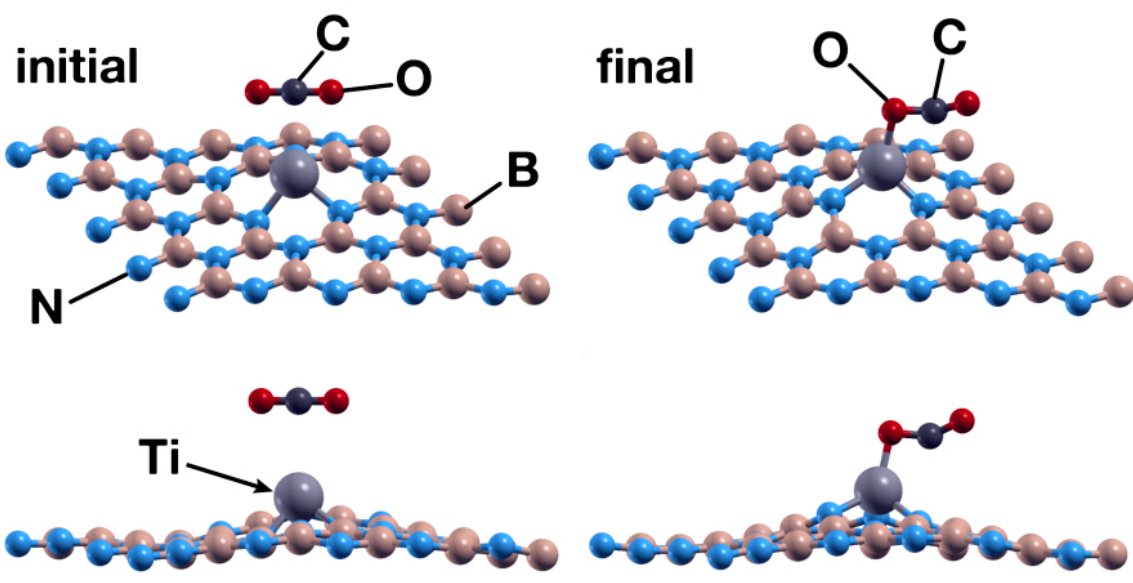

Figure 6. Initial (left) and final (right) configurations for the interaction of the hBN surface with a Ti absorbed in a B vacancy and a $\mathrm{CO}_{2}$ molecule. As in Figure 4, the upper row shows a system's perspective, while the lower one presents the $\mathrm{XZ}$ plane. The titanium atom acts as a catalyst to chemisorb the $\mathrm{CO}_{2}$ molecule in the system. 
In Figure 7, we notice the hybridization of the $3 \mathrm{~d}$ orbital from Ti and the $p$ orbitals from $\mathrm{B}, \mathrm{N}, \mathrm{C}$, and $\mathrm{O}$ below $-2.5 \mathrm{eV}$ and above $+1.0 \mathrm{eV}$.

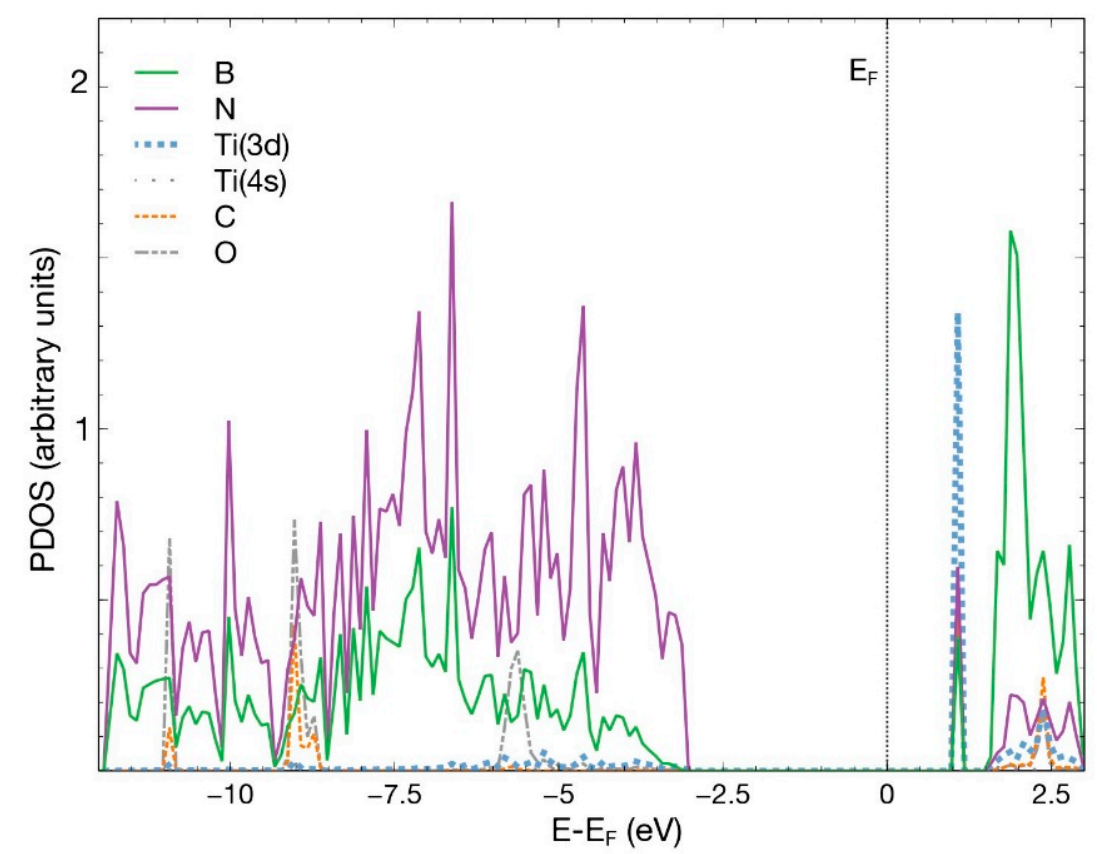

Figure 7. PDOS for the combined system $\mathrm{hBN}+\mathrm{Ti}$ and the adsorbed $\mathrm{CO}_{2}$ molecule. The plot shows the $p$ orbitals from all the B, N, C, O atoms and the $3 d$ and $4 s$ orbitals from Ti. The contribution from orbital $4 s$ from $\mathrm{Ti}$ is too small to be noticed in the figure.

\subsubsection{Pt Absorption on an hBN Surface with a B Vacancy}

As in the first case, we placed the platinum atom at $2.5 \AA$ above the surface, directly over the vacancy site. In the same way, and after 441 steps, the Pt atom chemisorbs with an absorption energy of $-6.93 \mathrm{eV}$ (Figure 8). The charge population analysis shows that the $\mathrm{Pt}$ atom donates 0.46 electrons to the surface, particularly to the $\mathrm{N}$ atoms surrounding it. Figure 9 shows the PDOS of the resulting system.
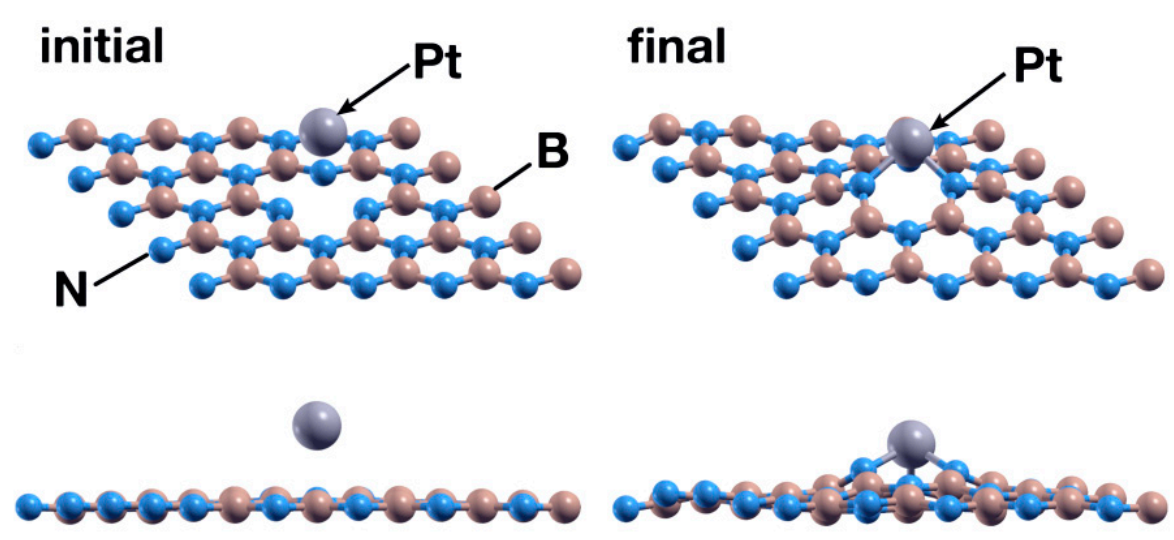

Figure 8. Initial (left) and final (right) configurations for the interaction of the hBN surface with a $B$ vacancy and a Pt atom. The Pt atom chemisorbs and deforms the surface slightly but more than the Ti atom does. 


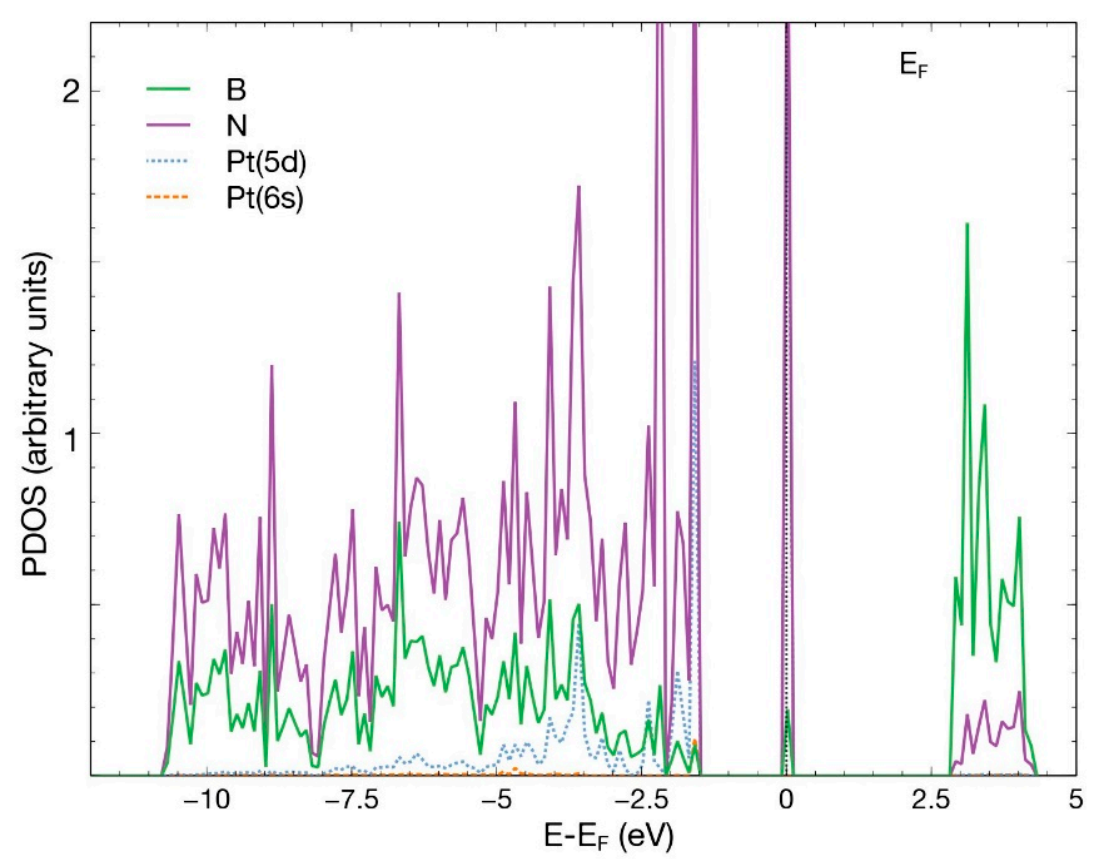

Figure 9. PDOS for the case in which a Pt atom chemisorbs in the vacancy site of an hBN surface with a boron vacancy. This figure presents the $p$ orbitals for boron and nitrogen and the orbitals $6 s$ and $5 d$ for Pt.

There is an evident hybridization of the $5 \boldsymbol{d}$ and $\mathbf{6 s}$ orbitals from Pt and $p$ orbitals from $\mathrm{B}$ and $\mathrm{N}$. The contribution of the $6 s$ orbital of $\mathrm{Pt}$ is small. A prominent and narrow peak appears around the Fermi energy.

\subsubsection{Interaction between $\mathrm{CO}_{2}$ and the $\mathrm{hBN}$ Surface $+\mathrm{Pt}$ in a B Vacancy}

The exploration of the interaction between a $\mathrm{CO}_{2}$ molecule and the system obtained in the previous case showed that the $\mathrm{Pt}$ atom is not a good enough catalyst for the absorption of the molecule. After 969 steps, the surface repels the $\mathrm{CO}_{2}$ molecule (see Figure 10).
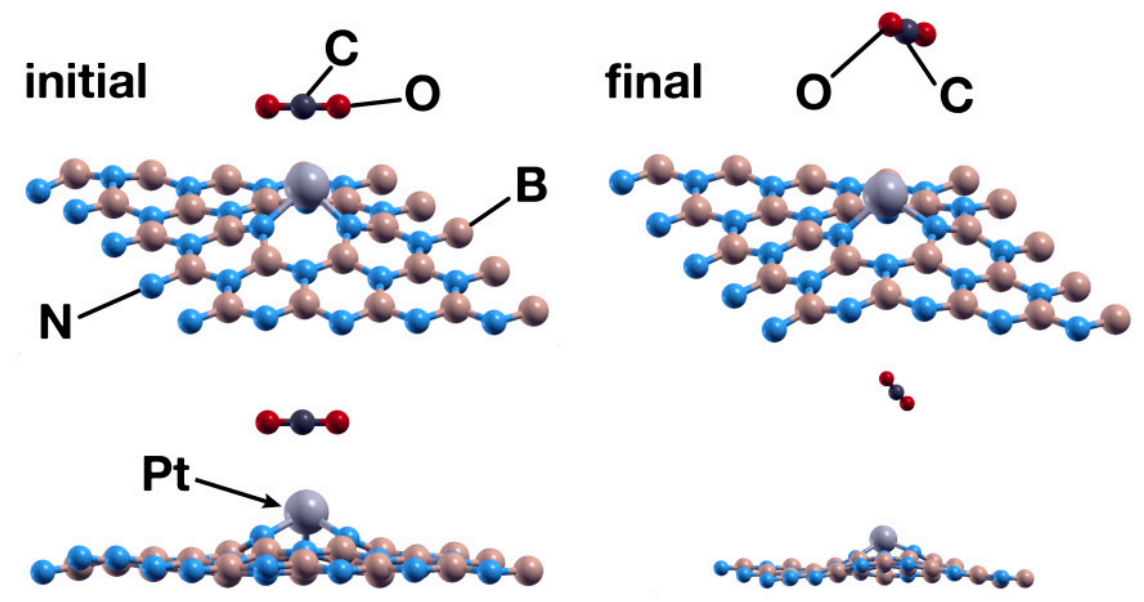

Figure 10. Initial (left) and final (right) configurations for the interaction of the hBN surface with a Pt absorbed in a $\mathrm{B}$ vacancy and a $\mathrm{CO}_{2}$ molecule. In this case, the system repels the $\mathrm{CO}_{2}$ molecule.

\section{2. hBN Surface with an N Vacancy}

We present this case in Figure 1b. From the PDOS shown in Figure 11, we can see that some states appear around the Fermi energy, and the hybridizations of $s$ and $p$ are below $-2.5 \mathrm{eV}$ and above $1.5 \mathrm{eV}$. 


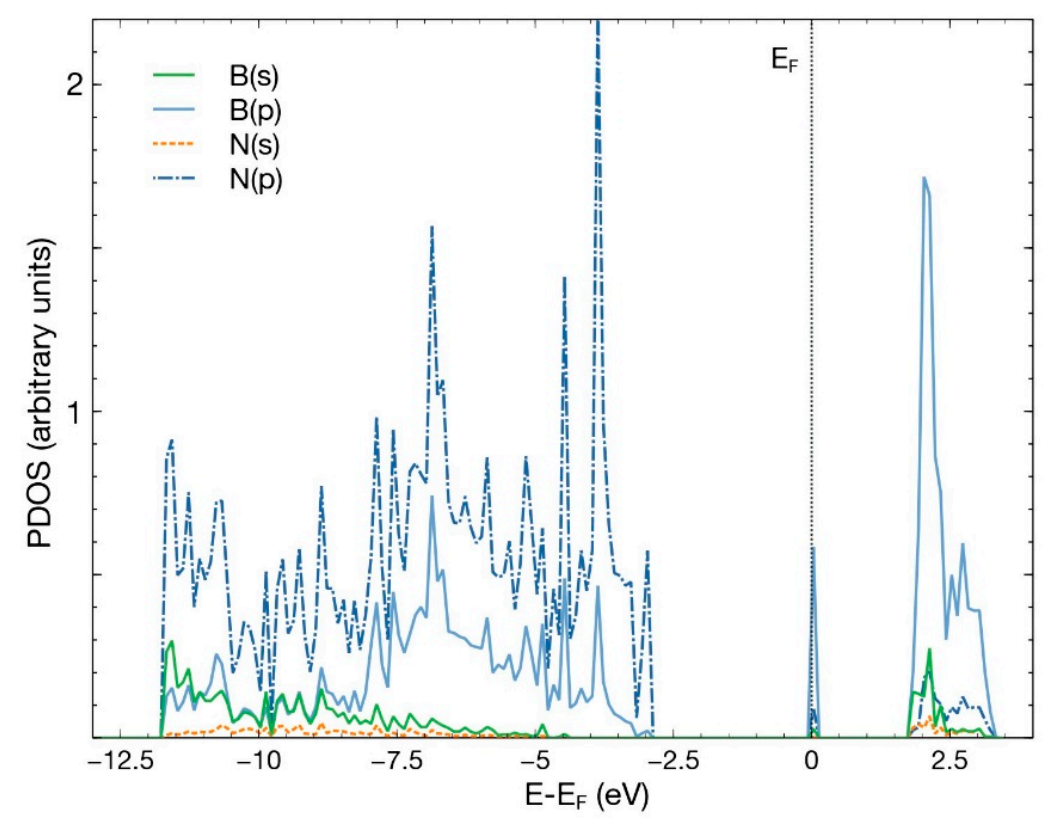

Figure 11. PDOS for the hBN surface with an $\mathrm{N}$ vacancy. The rearrangement of states and, therefore, of charge turns evident around the Fermi energy. The $s$ and $p$ orbitals of boron and nitrogen are shown.

\subsubsection{Ti Absorption on an N Vacancy}

We placed a Ti atom in conditions analogous to those of Section 3.1.1. After 1089 steps, the Ti atom chemisorbs at the vacancy site, with absorption energy of $-2.62 \mathrm{eV}$. Figure 12 shows the initial and final configuration for this case. There is a transfer of 0.89 electrons from Ti to its neighbors, less than the quantity observed in Section 3.1.1. This reduction may explain the smaller value of the absorption energy compared with the section above. Figure 13 shows the PDOS for this case. We can see a hybridization of the $4 s$ and $3 d$ orbitals from Ti with the $p$ orbitals from $\mathrm{B}$ and $\mathrm{N}$ below $-2.5 \mathrm{eV}$ and above $+2.5 \mathrm{eV}$.
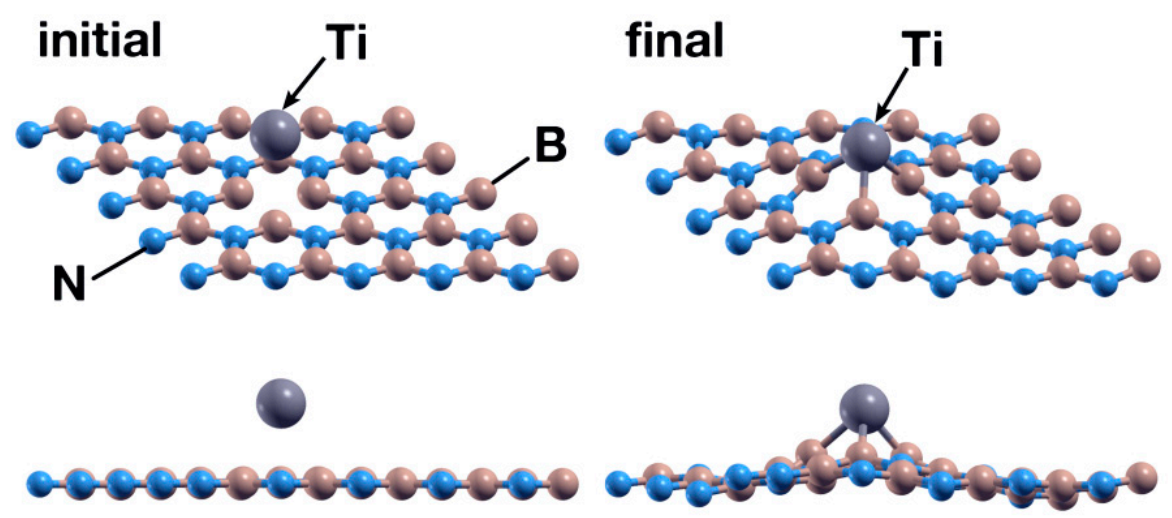

Figure 12. Initial (left) and final (right) configurations of the FPMD calculation for the second case considered. The upper images show a perspective view of the system, while the lower images show the $\mathrm{XZ}$ plane. The titanium atom chemisorbs in the hBN surface with an $\mathrm{N}$ vacancy, occupying the vacancy site. 


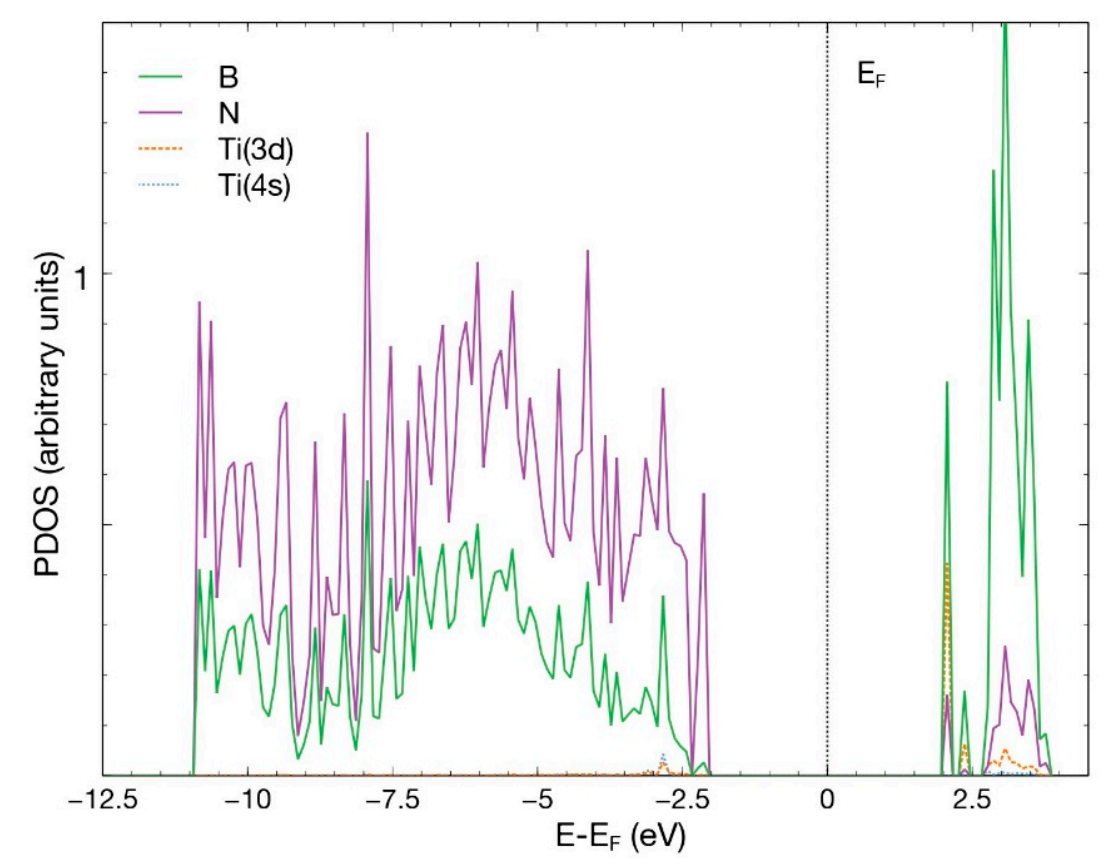

Figure 13. PDOS for the chemisorption of a Ti atom in an hBN surface with an $\mathrm{N}$ vacancy. We present $p$ orbitals from B and $\mathrm{N}$ and the $3 d$ and $4 s$ orbitals from Ti.

\subsection{2. $\mathrm{CO}_{2}$ Absorption on an $\mathrm{hBN}$ Surface + Ti Absorbed in an N Vacancy}

The Ti atom acts as a catalyst in this case as well. With the same initial orientation and vertical distance as the analogous case in Section 3.1.2, the $\mathrm{CO}_{2}$ molecule is chemisorbed by the surface, with energy of $-3.95 \mathrm{eV}$. However, there was a qualitative difference, as in this case, the $\mathrm{CO}_{2}$ molecule dissociated in two fractions: $\mathrm{CO}$ and $\mathrm{O}$. One oxygen atom bounds to a boron atom and the $\mathrm{Ti}$ atom, as shown in Figure 14. The $\mathrm{C}$ atom also bounds to the Ti atom. The Ti atom transfers 0.35 electrons to the $\mathrm{CO}_{2}$ molecule, and the $\mathrm{O}$ atom bound to boron and titanium received 0.23 electrons.

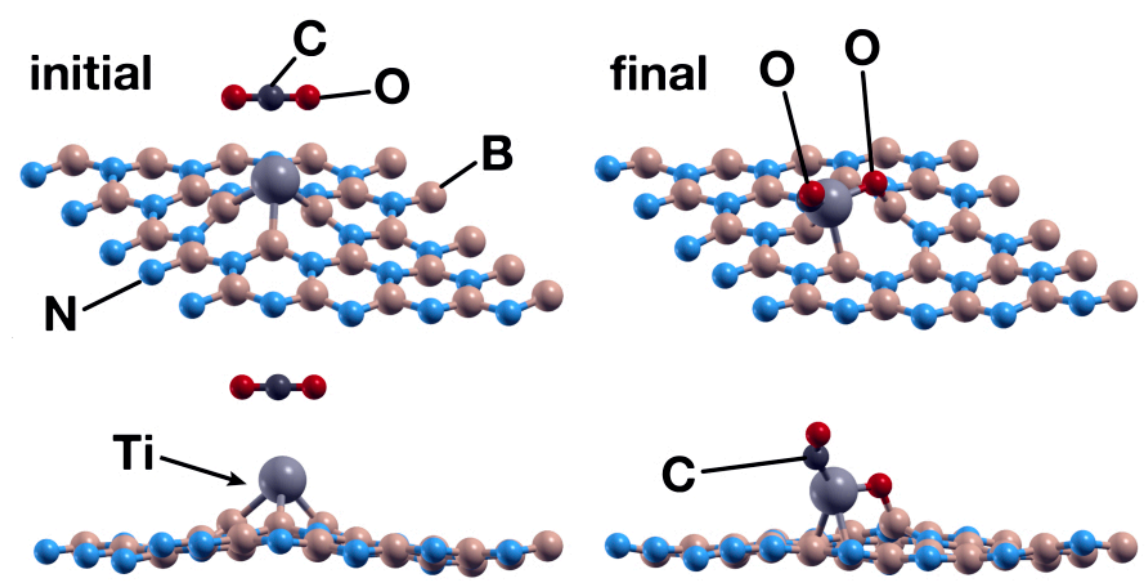

Figure 14. Initial (left) and final (right) configurations for the interaction of the hBN surface with a $\mathrm{Ti}$ absorbed in an $\mathrm{N}$ vacancy and a $\mathrm{CO}_{2}$ molecule. The titanium atom acts as a catalyst to chemisorb the $\mathrm{CO}_{2}$ molecule in the system.

Figure 15 shows the PDOS for this case. We notice a hybridization of the $3 \boldsymbol{d}$ orbital from titanium and the $p$ orbitals from boron, nitrogen, carbon, and oxygen, below $-2.5 \mathrm{eV}$ and above $+2.5 \mathrm{eV}$. The contribution from the $4 s$ orbital of $\mathrm{Ti}$ is minimal. 


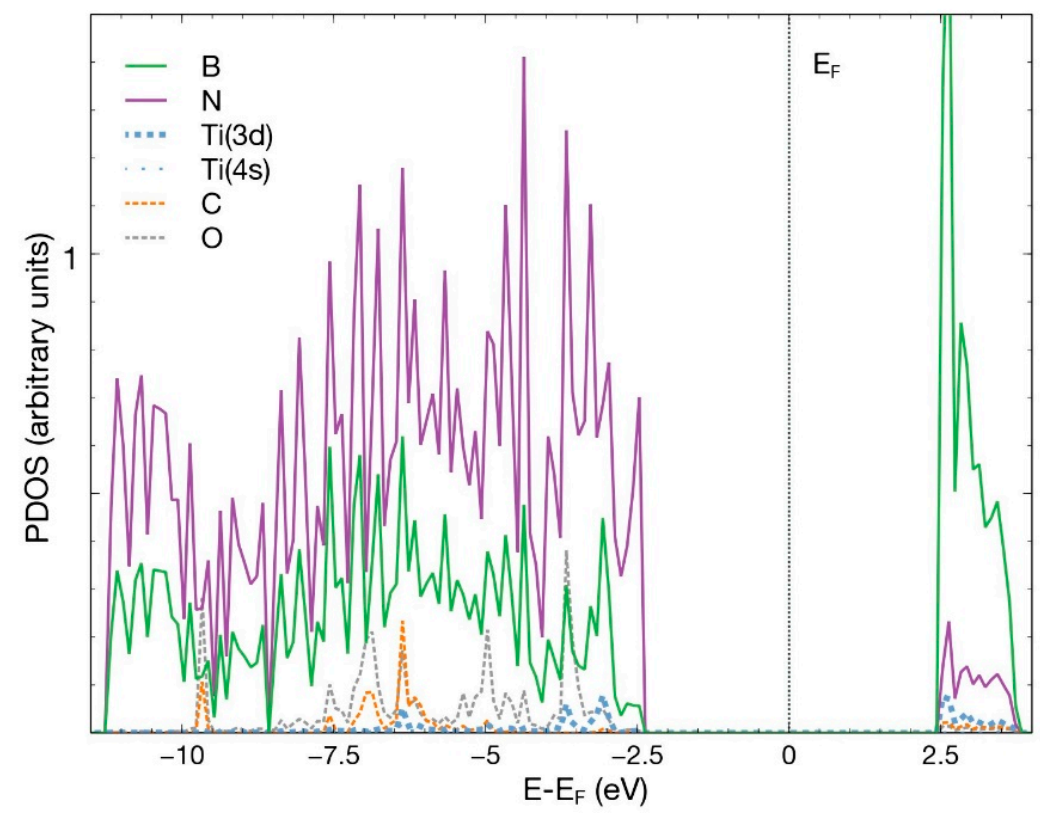

Figure 15. PDOS for the combined system of hBN with a Ti atom chemisorbed in an $\mathrm{N}$ vacancy and a molecule of $\mathrm{CO}_{2}$, which is chemisorbed and dissociated. The $p$ orbitals from boron, nitrogen, carbon, oxygen, and the $3 d$ and $4 s$ orbitals from titanium are shown.

\subsubsection{Pt Absorption on an hBN Surface with an $\mathrm{N}$ Vacancy}

The Pt atom, placed at $2.5 \AA$ above the surface, directly over the vacancy site, is also absorbed with an energy of $-6.59 \mathrm{eV}$. Figure 16 shows the initial and final configurations after 879 steps. The Pt atom donates 0.1 electrons to the surface, a smaller amount than the analogous case involving titanium (0.89) from Section 3.2.1. Figure 17 shows the PDOS of the combined system.
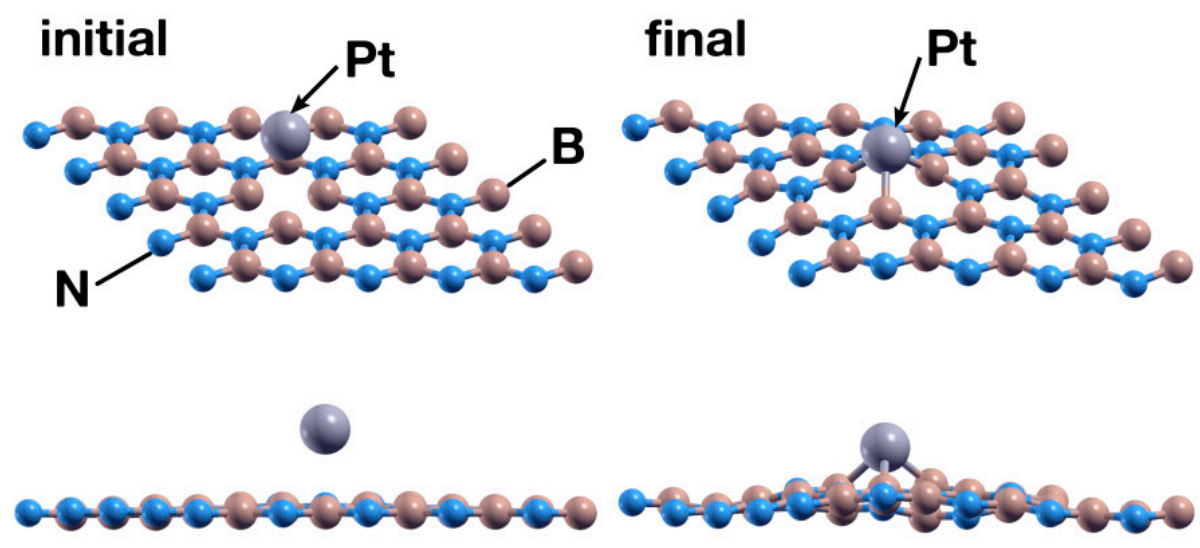

Figure 16. Initial (left) and final (right) configurations for the interaction of the hBN surface with an $\mathrm{N}$ vacancy and a Pt atom. The Pt atom is chemisorbed by the surface, just as it was in the hBN surface with a B vacancy. 


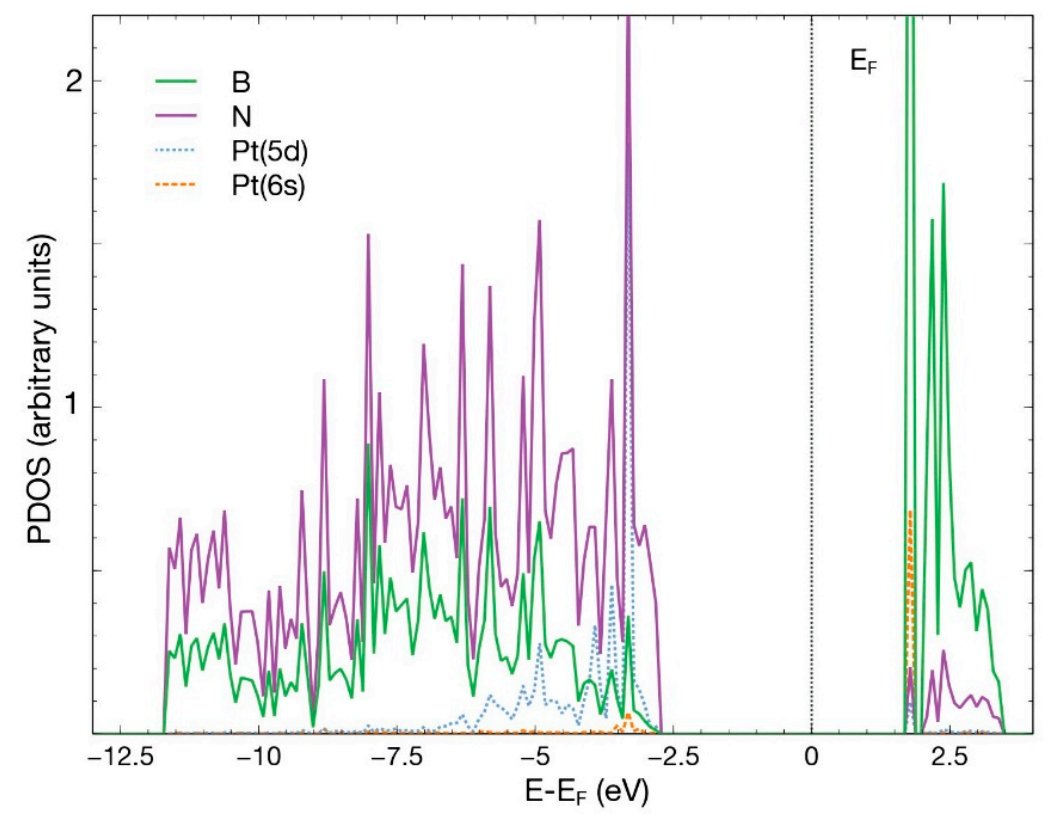

Figure 17. PDOS for the Pt atom chemisorbed on an N-vacancy site of the hBN surface.

Interaction between $\mathrm{CO}_{2}$ and the hBN Surface $+\mathrm{Pt}$ in an $\mathrm{N}$ Vacancy

The final case considered saw no interaction between the $\mathrm{CO}_{2}$ molecule and the surface. The MD run was 1894 steps long. We show the initial and final configurations in Figure 18. Table 1 summarizes the absorption energies or lack of interaction of the eight cases considered in this work.

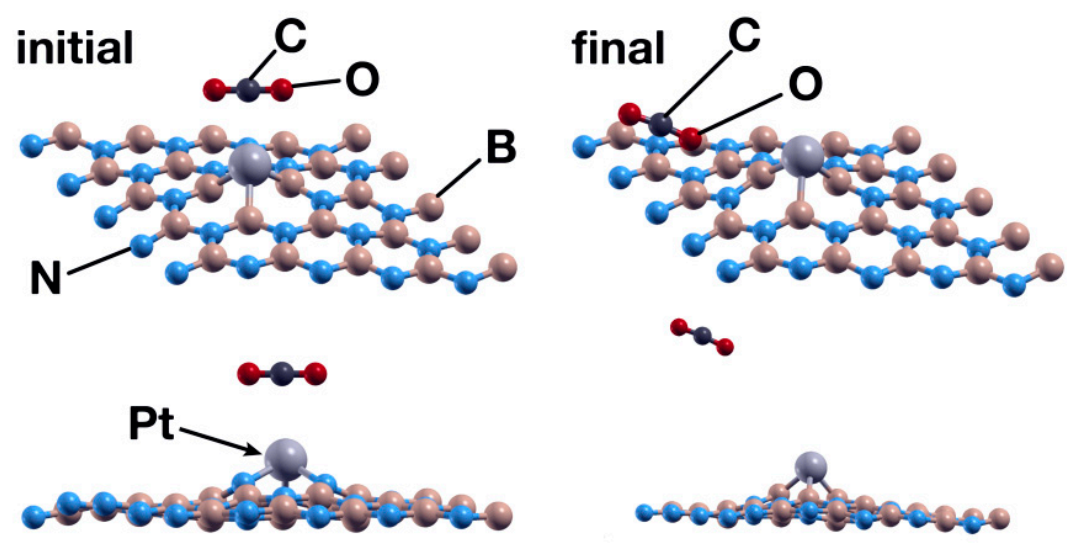

Figure 18. Initial (left) and final (right) configurations for the interaction of the hBN surface with a $\mathrm{Pt}$ absorbed in an $\mathrm{N}$ vacancy and a $\mathrm{CO}_{2}$ molecule. As in the case where the Pt atom chemisorbs in a $\mathrm{B}$ vacancy, the surface repels the molecule.

Table 1. Absorption energies (in eV) for the interaction between the hBN surface and a Ti atom or a $\mathrm{Pt}$ atom and the subsequent interaction with a $\mathrm{CO}_{2}$ molecule.

\begin{tabular}{cc}
\hline System & $\mathbf{E}_{\mathbf{a b s}}$ \\
\hline Ti absorbed on hBN with B vacancy & -11.39 \\
$\mathrm{CO}_{2}$ absorbed on hBN + Ti on B vacancy & -1.04 \\
Pt absorbed on hBN with B vacancy & -6.93 \\
$\mathrm{CO}_{2}$ absorbed on hBN + Pt on B vacancy & No absorption \\
$\mathrm{Ti}$ absorbed on hBN with N vacancy & -2.62 \\
$\mathrm{CO}_{2}$ absorbed on hBN + Ti on N vacancy & -3.95 \\
Pt absorbed on hBN with N vacancy & -6.59 \\
$\mathrm{CO}_{2}$ absorbed on hBN + Pt on N vacancy & No absorption \\
\hline
\end{tabular}




\section{Discussion}

We performed FPMD simulations at $300 \mathrm{~K}$ and atmospheric pressure. We found that the pristine hBN surface shows no interaction with the $\mathrm{CO}_{2}$ molecule, and this situation did not change with a vacancy either of a boron or a nitrogen atom. The titanium and platinum atoms chemisorb on the surface only in the presence of a vacancy. However, only the Ti atom could absorb the $\mathrm{CO}_{2}$ molecule. This fact is probably due to the amount of charge exchanged between the Ti atom and the hBN surface, in both cases considered. The number of exchanged electrons was more significant for Ti than for Pt always.

The absorption of the $\mathrm{CO}_{2}$ molecule occurs in two ways. One is without dissociation, and, in the other, the molecule breaks in $\mathrm{CO}$ and $\mathrm{O}$.

In the two cases where the $\mathrm{CO}_{2}$ chemisorbs on the $\mathrm{hBN}+\mathrm{Ti}$ surface, the energies are more significant than the standard free energy for gaseous $\mathrm{CO}_{2}$ at room temperature, which is $0.67 \mathrm{eV}$ [2]. This fact suggests that the combined hBN-Ti systems may capture $\mathrm{CO}_{2}$ at ambient conditions.

These results are consistent with previous studies we made, where we found that both the Ti and $\mathrm{Pt}$ atoms can absorb a CO molecule in similar conditions [27]. The more considerable amount of charge involved in the absorption process for the $\mathrm{CO}_{2}$ molecule may have inhibited the catalyst action of the Pt atom.

Author Contributions: Conceptualization, J.M.R.-d.-A., A.F.J.G. and L.F.M.; Data curation, J.M.R.d.-A. and A.F.J.G.; Formal analysis, J.M.R.-d.-A., A.F.J.G. and L.F.M.; Funding acquisition, L.F.M.; Investigation, J.M.R.-d.-A., A.F.J.G. and L.F.M.; Methodology, J.M.R.-d.-A., A.F.J.G. and L.F.M.; Project administration, L.F.M.; Resources, L.F.M.; Validation, J.M.R.-d.-A., A.F.J.G. and L.F.M.; Writingoriginal draft, J.M.R.-d.-A. and L.F.M.; Writing_review and editing, J.M.R.-d.-A. and L.F.M. All authors have read and agreed to the published version of the manuscript.

Funding: This research was funded by Dirección General de Asuntos del Personal Académico de la Universidad Nacional Autónoma de México, grant number IN113220. The APC was funded by Tecnologico de Monterrey.

Institutional Review Board Statement: Not applicable.

Informed Consent Statement: Not applicable.

Acknowledgments: We thank Dirección General de Asuntos del Personal Académico de la Universidad Nacional Autónoma de México, partial financial support by Grant IN113220. We also appreciate UNAM-Miztli-Super-Computing Center technical assistance by the project LANCADUNAM-DGTIC-030.

Conflicts of Interest: The authors declare no conflict of interest.

\section{References}

1. Watanabe, K.; Taniguchi, T.; Kanda, H. Direct-Bandgap Properties and Evidence for Ultraviolet Lasing of Hexagonal Boron Nitride Single Crystal. Nat. Mater. 2004, 3, 404-409. [CrossRef] [PubMed]

2. Tran, T.T.; Bray, K.; Ford, M.J.; Toth, M.; Aharonovich, I. Quantum Emission from Hexagonal Boron Nitride Monolayers. Nat. Nanotechnol. 2016, 11, 37-41. [CrossRef]

3. Ajori, S.; Ameri, A.; Ansari, R. Characterizing the Mechanical Properties and Fracture Pattern of Defective Hexagonal BoronNitride Sheets with Focus on Stone-Wales Defect. Superlattices Microstruct. 2020, 142, 106526. [CrossRef]

4. Rahman, F.A.; Aziz, M.M.A.; Saidur, R.; Bakar, W.A.W.A.; Hainin, M.R.; Putrajaya, R.; Hassan, N.A. Pollution to Solution: Capture and Sequestration of Carbon Dioxide $\left(\mathrm{CO}_{2}\right)$ and Its Utilization as a Renewable Energy Source for a Sustainable Future. Renew. Sustain. Energy Rev. 2017, 71, 112-126. [CrossRef]

5. Choi, H.; Park, Y.C.; Kim, Y.-H.; Lee, Y.S. Ambient Carbon Dioxide Capture by Boron-Rich Boron Nitride Nanotube. J. Am. Chem. Soc. 2011, 133, 2084-2087. [CrossRef] [PubMed]

6. Gao, G.; Ma, F.; Jiao, Y.; Sun, Q.; Jiao, Y.; Waclawik, E.; Du, A. Modelling $\mathrm{CO}_{2}$ Adsorption and Separation on ExperimentallyRealized B 40 Fullerene. Comput. Mater. Sci. 2015, 108, 38-41. [CrossRef]

7. Sun, Q.; Li, Z.; Searles, D.J.; Chen, Y.; Lu, G.; Du, A. Charge-Controlled Switchable $\mathrm{CO}_{2}$ Capture on Boron Nitride Nanomaterials. J. Am. Chem. Soc. 2013, 135, 8246-8253. [CrossRef] [PubMed]

8. Qu, M.; Qin, G.; Du, A.; Fan, J.; Sun, Q. B 80 Fullerene: A Promising Metal-Free Photocatalyst for Efficient Conversion of $\mathrm{CO}_{2}$ to HCOOH. J. Phys. Chem. C 2019, 123, 24193-24199. [CrossRef] 
9. Owuor, P.S.; Park, O.-K.; Woellner, C.F.; Jalilov, A.S.; Susarla, S.; Joyner, J.; Ozden, S.; Duy, L.; Villegas Salvatierra, R.; Vajtai, R.; et al. Lightweight Hexagonal Boron Nitride Foam for $\mathrm{CO}_{2}$ Absorption. ACS Nano 2017, 11, 8944-8952. [CrossRef]

10. Wang, L.; Wang, Y.; Zhang, R.; Ding, R.; Chen, X.; Lv, B. Edge-Activating $\mathrm{CO}_{2}-$ Mediated Ethylbenzene Dehydrogenation by a Hierarchical Porous BN Catalyst. ACS Catal. 2020, 10, 6697-6706. [CrossRef]

11. Parr, R.G.; Yang, W. Density-Functional Theory of Atoms and Molecules; International Series of Monographs on Chemistry; Oxford University Press: Oxford, UK; Clarendon Press: New York, NY, USA, 1989; ISBN 978-0-19-504279-5.

12. Harrison, W.A. Pseudopotentials in the Theory of Metals; Frontiers in Physics; University of Virginia: Charlottesville, VA, USA, 1966.

13. Giannozzi, P.; Baroni, S.; Bonini, N.; Calandra, M.; Car, R.; Cavazzoni, C.; Ceresoli, D.; Chiarotti, G.L.; Cococcioni, M.; Dabo, I.; et al. Quantum Espresso: A Modular and Open-Source Software Project for Quantum Simulations of Materials. J. Phys. Condens. Matter. 2009, 21, 395502. [CrossRef] [PubMed]

14. Giannozzi, P.; Andreussi, O.; Brumme, T.; Bunau, O.; Buongiorno Nardelli, M.; Calandra, M.; Car, R.; Cavazzoni, C.; Ceresoli, D.; Cococcioni, M.; et al. Advanced Capabilities for Materials Modelling with Quantum ESPRESSO. J. Phys. Condens. Matter. 2017, 29, 465901. [CrossRef]

15. Kokalj, A. XCrySDen-A New Program for Displaying Crystalline Structures and Electron Densities. J. Mol. Graph. Model. 1999, 17, 176-179. [CrossRef]

16. Troullier, N.; Martins, J.L. Efficient Pseudopotentials for Plane-Wave Calculations. Phys. Rev. B 1991, 43, 1993-2006. [CrossRef]

17. Blöchl, P.E. Projector Augmented-Wave Method. Phys. Rev. B 1994, 50, 17953-17979. [CrossRef] [PubMed]

18. Kleinman, L.; Bylander, D.M. Efficacious Form for Model Pseudopotentials. Phys. Rev. Lett. 1982, 48, 1425-1428. [CrossRef]

19. Perdew, J.P.; Burke, K.; Ernzerhof, M. Generalized Gradient Approximation Made Simple. Phys. Rev. Lett. 1996, 77, 3865, reprinted in Phys. Rev. Lett. 1997, 78, 1396-1396. [CrossRef]

20. Lindan, P.J.D.; Harrison, N.M.; Gillan, M.J.; White, J.A. First-Principles Spin-Polarized Calculations on the Reduced and Reconstructed $\mathrm{TiO}_{2}$ (110) Surface. Phys. Rev. B 1997, 55, 15919-15927. [CrossRef]

21. Monkhorst, H.J.; Pack, J.D. Special Points for Brillouin-Zone Integrations. Phys. Rev. B 1976, 13, 5188-5192. [CrossRef]

22. Oura, K. (Ed.) Surface Science: An Introduction; Advanced Texts in Physics; Springer: Berlin, Germany; New York, NY, USA, 2003; ISBN 978-3-540-00545-2.

23. Pease, R.S. An X-ray Study of Boron Nitride. Acta Cryst. 1952, 5, 356-361. [CrossRef]

24. Lide, D.R. (Ed.) CRC Handbook of Chemistry and Physics: A Ready-Reference Book of Chemical and Physical Data, 81st ed.; CRC Press: Boca Raton, FL, USA, 2000; ISBN 978-0-8493-0481-1.

25. Novoselova, T.; Malinov, S.; Sha, W.; Zhecheva, A. High-Temperature Synchrotron X-Ray Diffraction Study of Phases in a Gamma TiAl Alloy. Mater. Sci. Eng. A 2004, 371, 103-112. [CrossRef]

26. Ochi, M.; Yamada, I.; Ohgushi, K.; Kusano, Y.; Mizumaki, M.; Takahashi, R.; Yagi, S.; Nishiyama, N.; Inoue, T.; Irifune, T. B-Site Deficiencies in A -Site-Ordered Perovskite LaCu 3 Pt 3.75 O12. Inorg. Chem. 2013, 52, 3985-3989. [CrossRef] [PubMed]

27. Ramirez-de-Arellano, J.M.; Magana-Solis, L.F. Interaction of CO with an HBN Surface Doped with Ti and Pt: A First Principles Study. J. Phys. Conf. Ser. 2017, 792, 012074. [CrossRef] 